\title{
Scattering Problem Modelling in IoT Antennas
}

\author{
Auns Khaled ${ }^{1, *}$, Dorsaf Omri ${ }^{1}$, and Taoufik Aguili $^{2}$ \\ ${ }^{1,2}$ Engineering School of Tunis, Tunisia \\ *Corresponding author, email: auns.khaled@enit.utm.tn
}

\begin{abstract}
Modern radio system requirements have pushed antenna theory and practice in novel directions. One of them investigates the performance of non-linear elements in antennas. Numerous factors influence the growth of leaders. For this This article describes a new technique for simulating the scattering problem in IoT antennas. The software uses the spatiotemporal integrative equation to predict the antenna's transient response (the electric field integrative equation). Laguerre functions provide a time basis. We use the RWG functions to solve an electric field integrative equation in the $2 \mathrm{D}$ time domain using a new hexagonal lattice approach (Rao, Wilton and Glisson function). This is due to the use of TD-IE to investigate the transient radiation from an Internet of Things (IoT) antenna illuminated by an incoming electromagnetic pulse. The brief radiation from an IoT antenna is investigated using a newly developed core function from RWG TD-IE. As well as Laguerre functions, a unique spatial basis function derived from RWG functions is used. The data results acquired support our plan. Digital data is incoherent.
\end{abstract}

Keywords: Hexahedral-Mesh; IOT antenna; Laguerre-Functions; Moments-Method; RWG functions; Time Domain Integral Equation

\section{INTRODUCTION}

The increasing requirements of modern radio and television systems have led to the creation of new approaches in antenna theory and practice. One of them is the study of the performance of antennas with non-linear elements. Several factors contribute to the trend's growth [1]. The first is a comprehensive introduction to practically active integrated antennas, based on the principles of integration of the active device and the radiator. In
[2], the authors stated that the application of antennas such as radiolocation, communications, wireless power transmission systems, radio frequency identification systems, and spatial power integration systems, allowed to solve a number of problems related to reducing antenna dimensions and improving its power characteristics. As a result, new types of antennas appeared, the operation of which is mainly based on the integration of NonLinear Elements (NLE). They include antenna rectifiers, frequency multiplexing antennas, mixing antennas, and antenna generators [3]. The acquisition of additional frequency bands and the widespread use of developments in the field of microelectronics continue to expand the field of applications of active integrated antennas.

The second aspect is the escalation of EMC concerns due to the increased sensitivity of radio transceivers. This circumstance necessitates investigating nonlinear adverse effects in ANE, most notably pseudo addiction. The spurious radiation can be produced by nonlinear elements (NE) necessary for antenna operation and parasitic nonlinearity. The antenna architecture can induce the latter (for example, the oxidized coatings formed at the antenna element junctions exhibit nonlinear voltampere properties) or by the unfavorable operating system of the active phased antenna array elements [4]. The growing interest in nonlinear effects research results from the emergence of nonlinear radiative determination techniques that use the fundamental harmonic frequency produced by the current element as an information signal. Nonlinear elements that change the spectral structure of the re-radiated field of an object are classified as intrinsic or parasitic nonlinear. Whether the antenna generates nonlinear effects through embedded NEs or parasitic nonlinearities, both types of devices must be viewed as one class 
of nonlinear antennas [5]. In [6], the authors presented alternative modelling methods that have grown in importance in the antenna structure design. The main reason for this is the high cost of analyzing a full-wave electromagnetic (EM) antenna system. This is important to ensure the reliability of the assessments, but it is costly. In [7], the authors presented iterative simulations are required for EMdriven design activities such as geometry parameter change or uncertainty quantification. Some design approaches, such as global research or throughput optimization, can be expensive at the simulation model level. Although accurate modelling of antenna structures is complex, quick-replacement (or replacement) models may help. As the number of antenna parameters increases, the number of training data samples needed to build a viable model increases proportionally. Performance-dependent modelling is a relatively new concept that focuses on a small subset of the parameter space that contains high-quality performance architectures [8]. The model field and the alternative are generated using kriging's interpolation in nested kriging. Field reservation improves model prediction capabilities while using a small number of training data samples by utilizing a set of pre-optimized reference designs [9].

The paper is divided into sections. The first section introduces the problem mathematically. The second section compares the numerical results of a transient plane wave scattering with a different base function use case. The third section ended the paper.

\section{MATHEMATICAL FORMULATION}

We take an arbitrary surface $\mathrm{S}$ energized by a transient electromagnetic wave Einc(t) to simulate a two-dimensional problem. Assume that the shape's surface is an ideal conductor. At the conductor's surface, the following conditions significantly:

$$
\overrightarrow{\mathrm{n}} \times \overrightarrow{\mathrm{E}^{\mathrm{mc}}}+\overrightarrow{\mathrm{n}} \times \overrightarrow{\mathrm{E}^{\mathrm{sca}}}=0
$$

Where $\left(\overrightarrow{\mathrm{E}^{\mathrm{Inc}}}\right)$ denotes the incident field, $\left(\overrightarrow{\mathrm{E}^{\text {sca }}}\right)$ denotes the scattered field, and $\mathrm{n}$ indicates the surface normal. The scattered field is defined in terms of both vector and scalar potentials [10]:

$\mathrm{E}^{\mathrm{sca}}(\mathrm{r}, \mathrm{t})=-\frac{\partial}{\partial \mathrm{t}} \mathrm{A}(\mathrm{r}, \mathrm{t})-\nabla \varphi(\mathrm{r}, \mathrm{t}) ; \forall \mathrm{r} \in \mathrm{S}$

The vector potential is given by $A(r, t)$. The scalar potential $\varphi(r, t)$ is defined as follows. We establish a new source vector $\mathrm{c}(\mathrm{r}, \mathrm{t})$ that we refer to as the Hertz vector [11] to handle the time derivative of the vector potential defined analytically by:

$$
\overrightarrow{\mathrm{J}(\mathrm{r}, \mathrm{t})}=\frac{\partial}{\partial \mathrm{t}} \overrightarrow{\mathrm{c}(\mathrm{r}, \mathrm{t})}
$$

Equations (1) and (2) have been combined to give a Time-Domain Integral Equation for Electric Fields (TD-EFIE). The following section will discuss our concerns about spatial resolution.

\subsection{Space Resolution: RWG Functions}

We solved the TD-EFIE using the mother technique's two-dimensional formula [12]. Indeed, we demonstrate the space-based functions employed to connect the structure. The following are the RWG functions:

$$
\mathrm{f}_{\mathrm{n}}^{ \pm}(\mathrm{r})=\left\{\begin{array}{l}
\frac{\mathrm{l}_{\mathrm{n}}}{2 \mathrm{~A}_{\mathrm{n}}^{+}} \rho_{\mathrm{n}}^{ \pm}, r \in \mathrm{T}_{\mathrm{n}}^{-} \\
0, \text { otherwise }
\end{array}\right.
$$

$A_{n}^{ \pm}$and $l_{n}$ denote the area of the triangles $T_{n}^{ \pm}$and the length of the joint edge, respectively. $\rho_{n}^{ \pm}$is the position vector of the point $r$ relative to the triangles $\mathrm{T}_{\mathrm{n}}^{ \pm}$. Free vertex. In Figure. $1, \mathrm{~T}_{\mathrm{n}}^{+}$and $\mathrm{T}_{\mathrm{n}}^{-}$are depicted. Fig.1 illustrates the triangle's pair $\mathrm{T}_{\mathrm{n}}^{+}$and $\mathrm{T}_{\mathrm{n}}^{-}$.

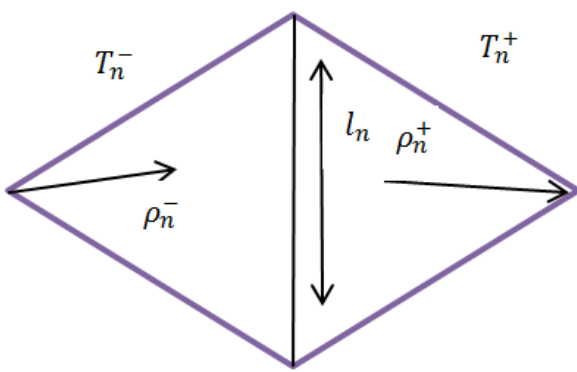

Figure 1. RWG function Representation.

Using spatial basis functions, we estimate the surface-induced current:

$$
C(r, t)=\sum_{n=1}^{N} C_{n}(t) f_{n}(r)
$$

Where, the number $\mathrm{N}$ denotes the domains space number. The following section shall see how the space basis function (4) is expressed in polar coordinates. 


\subsection{Polar Coordinate}

The polar coordinate is used to simplify the TDEFIE equation. Notably, if the observation point is $\mathrm{p}($,$) and the source point is p' (','). The position$ vectors $r$ and $r$ ' indicate the observation and source positions. For this reason, the following formula [13] denotes rr'$^{\prime}$

$r r^{\prime}=\sqrt{\rho^{2}+\rho^{\prime 2}-2 \rho \rho^{\prime} \cos \left(\theta-\theta^{\prime}\right)}$

It is expressed in the polar system. So, we use MoM to resolve TD-EFIE. Thus, $\mathrm{f} n$ represents the unknown Hertz vector (3). (r). Then we project the TD-EFIE into space to get:

$$
\begin{aligned}
<\mathrm{f}_{\mathrm{m}(\mathrm{r}), \frac{\partial}{\partial \mathrm{t}} \mathrm{A}(\mathrm{r}, \mathrm{t})>} & +<\mathrm{f}_{\mathrm{m}}(\mathrm{r}), \nabla \varphi(\mathrm{r}, \mathrm{t})>=< \\
\mathrm{f}_{\mathrm{m}}(\mathrm{r}), \mathrm{E}^{\mathrm{inc}}(\mathrm{r}, \mathrm{t})> & \\
& \forall \mathrm{m}=1 \ldots, \mathrm{N}
\end{aligned}
$$

Considering that $[$ Amnpq] $=$ anmpq is the space matrix ( $p$ and $q$ are positive or negative), where:

$\mathrm{a}_{\mathrm{nm}}^{\mathrm{pq}}=$

$\frac{1}{2 \pi} \int_{\rho(\mathrm{n})}^{\rho(\mathrm{n}+1)} \int_{\theta(\mathrm{n})}^{\theta(\mathrm{n}+1)} \cdot \rho_{\mathrm{n}}^{\mathrm{q}} \int_{\rho(\mathrm{m})}^{\rho(\mathrm{m}+1)} \int_{\theta(\mathrm{m})}^{\theta(\mathrm{m}+1)} \frac{\rho_{\mathrm{m}}^{\mathrm{p}}}{\mathrm{R}} \mathrm{d} \rho^{\prime} \mathrm{d} \rho \mathrm{d} \theta^{\prime} \mathrm{d} \theta$

On the other hand, the matrix [B_mn $\left.{ }^{\wedge} \mathrm{pq}\right]$ $=\mathrm{b} \_n m^{\wedge} \mathrm{pq}$ is noted with:

$\mathrm{b}_{\mathrm{mn}}^{\mathrm{pq}}=$

$\frac{1}{4 \pi} \int_{\rho(\mathrm{n})}^{\rho(\mathrm{n}+1)} \int_{\theta(\mathrm{n})}^{\theta(\mathrm{n}+1)} \frac{\mathrm{l}_{\mathrm{n}}^{2}}{\mathrm{~A}_{\mathrm{n}}^{+}} \int_{\rho(\mathrm{m})}^{\rho(\mathrm{m}+1)} \int_{\theta(\mathrm{m})}^{\theta(\mathrm{m}+1)} \frac{1}{\mathrm{R}} \frac{\mathrm{l}_{\mathrm{n}}^{2}}{\mathrm{~A}_{\mathrm{n}}^{-}} \mathrm{d} \rho^{\prime} \mathrm{d} \rho \mathrm{d} \theta^{\prime} \mathrm{d} \theta$

The divergence's expression is explained in [14]. The integral's bonds are depicted in Figure 2. Finally, we arrive at the following equation:

$\sum_{\mathrm{n}=1}^{\mathrm{N}} \sum_{\mathrm{p}, \mathrm{q}}\left[\mu \mathrm{a}_{\mathrm{mn}}^{\mathrm{pq}} \frac{\mathrm{d}^{2}}{\mathrm{dt}^{2}} \mathrm{c}_{\mathrm{n}}\left(\tau_{\mathrm{mn}}^{\mathrm{pq}}\right)+\frac{\mathrm{b}_{\mathrm{mn}}^{\mathrm{pq}}}{\varepsilon} \mathrm{c}_{\mathrm{n}}\left(\tau_{\mathrm{mn}}^{\mathrm{pq}}\right)\right]=$

$\mathrm{V}_{\mathrm{m}}^{\mathrm{E}}(\mathrm{t})$

$V_{m}^{E}(r, t)=\int f_{m}(r) \cdot E^{i n c}(r, t) d S$

Where c denotes the electromagnetic wave's propagation velocity $\mu$, and $\varepsilon$ denote the medium's permeability and permittivity. The structure of the IoT antenna is depicted in Figure 2.

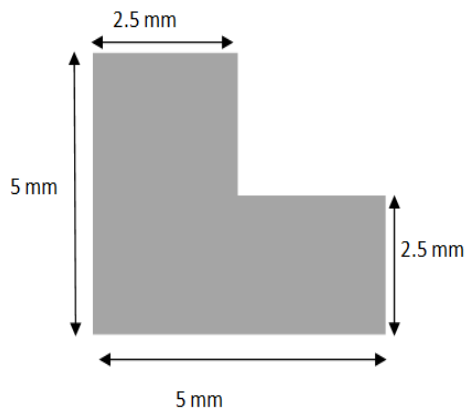

Figure 2: Meshing structure

The following section discusses the problem's time resolution via a temporal basis function.

\subsection{Temporal resolution}

The following section discusses the problem's time resolution via a temporal basis function.

$$
c_{n}(t)=\sum_{i=0}^{N} c_{n, i} \varphi_{i}(s t)
$$

\section{NUMERICAL RESULTS}

This section provides the numerical results produced by employing the hexagonal technique mesh with the method stated previously. We assume a conducting patch with a radius of $0.001 \mathrm{~m}$ and a length of $1 \mathrm{~m}$. A transient wave in free space excites the patch antenna as follows:

$E^{\text {inc }}(t)=\left\{\begin{array}{l}1,10^{-9} s \leq t \leq 10^{-8} s \\ 0, \text { otherwire }\end{array}\right.$

The observation interval $[0,1.4 * 10(-7) \mathrm{s}]$ is divided into $\mathrm{t}=0.7 * 10(-9)$ subdomains. The Laguerre parameters are $\mathrm{M}$ and the scaling factor $« \mathrm{~s} »$. As shown in $[10,12], \mathrm{M}=80$ and $\mathrm{s}=7.068 * 108$ are sufficient for accurate results. As shown in Figure 3, the spatial distribution is horizontally polarized.

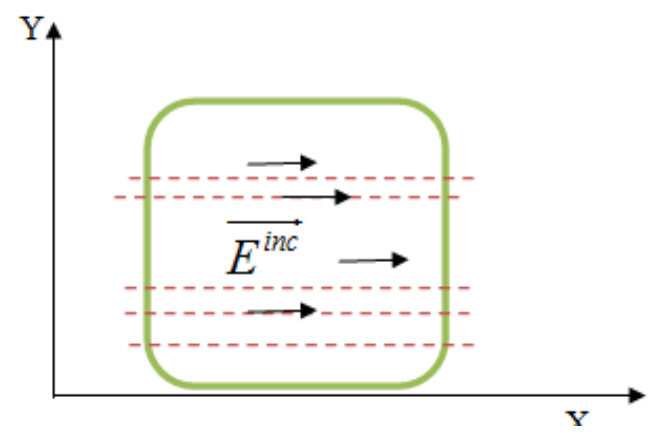

Figure 3. Polarization of the incident electric field We compare the results obtained using the innovative method mesh to those obtained using a 
triangle mesh to validate the method mesh. We reconstruct the preceding formulation using the triangular mesh technique. As a result, the result depicted in Figure 4 is obtained. For $\mathrm{N}=16$, we conclude that hexagonal meshes outperform triangular meshes.

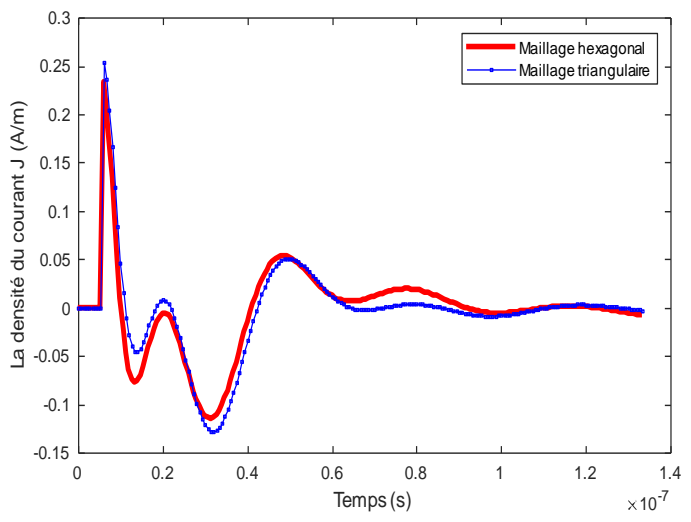

Figure 4: Current transient response (obtained by two different type of mesh)

The density distribution that exists at the moment is described. The current space density is depicted in Figure 5 at $\mathrm{t} 1=1.933310-8 \mathrm{~s}, \mathrm{t} 2=3.266710-8 \mathrm{~s}$, and $\mathrm{t} 3=6.600010-8$ s. The current amplitude is significant initially because it explains transitory events. Thus, the spatial distribution of the injected current density illustrates the transient system's appearance over time.

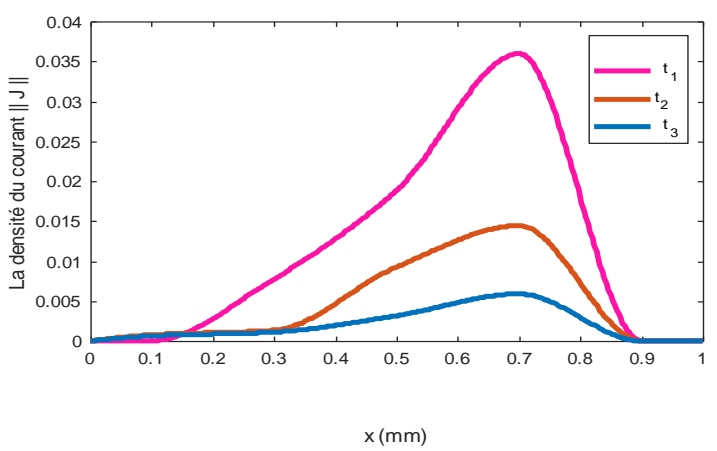

Figure 5: Current Spatial distribution

Additionally, the table1 displays the computed CPU-Time (simulation time) for the triangular and hexagonal meshes. As a result, we observe that hexagonal meshes perform better CPU time than triangular meshes do.
Table 1. CPU-Time.

\begin{tabular}{|c|c|}
\hline Time (s) & Mesh technique \\
\hline $\mathbf{9 3 . 6}$ & Triangular \\
\hline $\mathbf{0 . 1 2 6}$ & Hexagonal \\
\hline
\end{tabular}

\section{CONCLUSION}

The mathematical model of the cut-off nonlinear antenna has been selected as an attractive research topic for industry and academia. As a result, many algorithms and software packages have been developed, and their efficiency has been verified by analyzing the performance of many types of ANE: antenna rectifiers (rectennas), nonlinear effects in active PAAs, antenna multipliers, and general properties (attributes) of nonlinear radar objects. In this vein, we addressed the transient scattering concerns in this paper by employing the mom to solve the time domain integral equation (TD-IE). Indeed, the impedance is critical for noise reduction with the linked antennas. We developed a twodimensional task to address the scattering of a disturbing wave on a rectangle patch to foresee the transitory response of the previous one. Then we applied Galerkin's to Methods of Moments, with RWG as the spatial basis and Laguerre as the temporal basis. To achieve the optimum result, we developed a novel hexagonal meshing technique. This technique applies to two-dimensional structures. Additionally, the mesh can be utilized to address fractal antennas used in broadband applications.

\section{REFERENCES}

[1] Bihari, S. Tripathi and A. Deepak, "Iterative weighted EM and iterative weighted $\mathrm{EM}^{\prime}$-index for scientific assessment of scholars", Scientometrics, vol. 126, no. 7, pp. 5551-5568, 2021. Available: 10.1007/s11192-021-03937-8.

[2] F. Chietera, R. Colella and L. Catarinucci, "Dielectric Resonators Antennas Potential Unleashed by 3D Printing Technology: A Practical Application in the IoT Framework", Electronics, vol. 11, no. 1, p. 64, 2021. Available: 10.3390/electronics11010064

[3] F. Chietera, R. Colella and L. Catarinucci, "Dielectric Resonators Antennas Potential Unleashed by 3D Printing Technology: A Practical Application in the IoT Framework", Electronics, 
vol. 11, no. 1, p. 64, 2021. Available: 10.3390/electronics 11010064.

[4] H. Huang, "Evolution of Millimeter-Wave Antenna Solutions and Designs to Cellular Phones", IEEE Access, vol. 8, pp. 187615-187622, 2020. Available: 10.1109/access.2020.3027424.

[5] A. Kordzadeh, D. Holzmann, A. Binder, T. Moldaschl, J. Sturm and A. Roshanghias, "Miniaturized On-Chip NFC Antenna versus Screen-Printed Antenna for the Flexible Disposable Sensor Strips", IoT, vol. 1, no. 2, pp. 309-319, 2020. Available: 10.3390/iot1020018.

[6] R. Kumar C, "Non-linear Analysis Reinforced Concrete Flexure Members Using Elements with Axial - Shear Coupling", International Journal of Contemporary Research and Review, vol. 08, no. 06, $2017 . \quad$ Available: 10.15520/ijcrr/2017/8/05/184.

[7] A. Mushtaq, A. Rajawat and S. Gupta, "Design of Antenna Array Based Beam Repositioning for IoT Applications", Wireless Personal Communications, 2021. Available: 10.1007/s11277-021-09046-2.

[8] S. Radaev, "Mathematical Modeling of Heat and Mass Transfer in Heat Pipes in a One-Dimensional Formulation when Cooling Active Phased Antenna Arrays", International Journal of Mechanics, vol. 15, pp. 196-203, 2021. Available: 10.46300/9104.2021.15.23.

[9] S. Taravati and G. Eleftheriades, "Space-Time Medium Functions as a Perfect Antenna-MixerAmplifier Transceiver", Physical Review Applied, vol. 14, no. 5, 2020. Available: 10.1103/physrevapplied.14.054017.

[10] Evan Groesen and Andonowati, "Hamiltonian Boussinesq formulation of wave-ship interactions", Applied Mathematical Modelling, vol. 42, pp. 133-144, 2017. Available: 10.1016/j.apm.2016.10.018.

[11] M. Ghorbani and H. Ghorbaninejad, "A Novel Ultrawideband Gear-Shaped Dielectric Ring Resonator Antenna", Mathematical Problems in Engineering, vol. 2021, pp. 1-8, 2021. Available: 10.1155/2021/8069873.

[12] A. Kordzadeh, D. Holzmann, A. Binder, T. Moldaschl, J. Sturm and A. Roshanghias, "Miniaturized On-Chip NFC Antenna versus Screen-Printed Antenna for the Flexible Disposable Sensor Strips", IoT, vol. 1, no. 2, pp. 309-319, 2020. Available: 10.3390/iot1020018.

[13] G. Vardoyan, M. Skrzypczyk and S. Wehner, "On the quantum performance evaluation of two distributed quantum architectures", Performance
Evaluation, vol. 153, p. 102242, 2022. Available: 10.1016/j.peva.2021.102242.

[14] P. Yin, F. Han, J. Wang, C. Lu and H. Du, "Influence of coordinate system establishment error on tooth profile deviation measured by standard polar coordinate method on gear measuring center", Measurement, vol. 187, p. 110344, 2022. Available: 10.1016/j.measurement.2021.110344. 